\title{
Spontaneous Corneal Clearance Despite Dislocated Graft after Descemet's Stripping Automated Endothelial Keratoplasty
}

\author{
Aina Malindri Dasrilsyah ${ }^{1}$, Mae-Lynn Catherine Bastion², Then Kong Yong ${ }^{3}$ \\ ${ }^{1}$ Department of Ophthalmology, Universiti Kebangsaan Malaysia Medical Centre (UKMMC), Kuala Lumpur, Malaysia. \\ 2Department of Ophthalmology, Universiti Kebangsaan Malaysia Medical Centre (UKMMC), Kuala Lumpur, Malaysia. \\ ${ }^{3}$ Department of Ophthalmology, International Specialist Eye Centre Malaysia (ISEC), Kuala Lumpur, Malaysia.
}

\section{INTRODUCTION}

Corneal transplantation is a widely practiced surgical procedure. Over the past decade, lamellar techniques have been developed to replace penetrating keratoplasty (PK). Endothelial Keratoplasty (EK) combines fast visual rehabilitation and low risk profile and has therefore replaced penetrating keratoplasty in the treatment of corneal endothelial diseases such as endothelial dystrophies (Fuchs' endothelial dystrophy, congenital hereditary endothelial dystrophy, posterior polymorphous dystrophy), pseudophakic and aphakic bullous keratopathy, endothelial decompensation caused by intraocular surgery, trauma or glaucoma drainage devices, iridocorneal endothelial (ICE) syndrome and endothelial failure post penetrating keratoplasty. $1,2,3$

The concept of EK is based on the creation of a (nearly) normal corneal anatomy by positioning a donor disc comprising endothelium and Descemet Membrane (DM) (DM endothelial keratoplasty, DMEK) or endothelium, DM and posterior stroma (Descemet stripping automated endothelial keratoplasty, DSAEK) onto the back surface of the recipient cornea. ${ }^{4}$ Graft detachment or dislocation is a major cause of complication following EK procedures. Rarely the recipient cornea may clear within several weeks to months after EK despite the presence of a detached or dislocated graft. $5,6,7,8,9,10,11$ The exact mechanism of spontaneous corneal clearance remains unknown.

In the present report, we describe a case of spontaneous corneal clearance despite dislocated graft into posterior chamber without replacement of the graft after DSAEK.

\section{PRESENTATION OF CASE}

A 48-year-old gentleman was referred for Endothelial Keratoplasty (EK) due to bullous keratopathy after he underwent four previous retinal detachment surgeries and complicated cataract surgery in 2010 over his right eye. He complained of progressive vision loss for about few years after cataract operation of the right eye. His visual acuity on the first presentation was hand movement (HM) over the right eye and no perception to light (NPL) over the left eye. Slit-lamp examination of the right eye showed corneal oedema. The eye was aphakic. As the patient was highly myopic, it was decided that secondary implant was unnecessary. Biometry reconfirmed that he would be better off without an intraocular lens implant. The axial length was $32 \mathrm{~mm}$. After discussing treatment options, risks and benefits, the patient agreed to undergo Descemet's stripping automated endothelial keratoplasty (DSAEK) of the right eye.
Corresponding Author:

Mae-Lynn Catherine Bastion,

Universiti Kebangsaan Malaysia Medical Centre(UKMMC), Jalan Yaacob Latif-56000,

Cheras, Kuala Lumpur, Malaysia.

E-mail: maelynnb2003@yahoo.com

DOI: $10.14260 / j e m d s / 2020 / 428$

How to Cite This Article:

Dasrilsyah AM, Bastion MLC, Yong TK. Spontaneous corneal clearance despite dislocated graft after descemet's stripping automated endothelial keratoplasty. J. Evolution Med. Dent. Sci. 2020;9(27):19671969, DOI: 10.14260/jemds/2020/428

Submission 14-04-2020,

Peer Review 04-06-2020

Acceptance 10-06-2020,

Published 06-07-2020.

Copyright (c) 2020 JEMDS. This is an open access article distributed under Creative Commons Attribution License [Attribution 4.0 International (CC BY 4.0)] 
An uncomplicated DSAEK was performed on the right eye in March 2018 using a standard surgical technique. Briefly, with a reverse Sinskey hook, a circular portion of Descemet membrane is scored and stripped from the posterior stroma so a descemetorhexis is created and the central portion of Descemet membrane is removed from the eye. A temporal selfsealing $4.0 \mathrm{~mm}$ sclerocorneal incision is created with a microkeratome knife. After trephinating an $8.5 \mathrm{~mm}$ diameter DSAEK-graft from the pre-dissected corneoscleral rim, the tissue is folded into a Busin spatula. A plastic lens glide is carefully inserted into the anterior chamber of the recipient. The lenticule was pulled into the anterior chamber using a Tan's forceps. The glide is removed, the DSAEK-graft is unfolded and left with a complete air fill for 8 minutes, after which the anterior chamber was reformed with Basic Salt Solution with a remaining $50 \%$ air fill. The patient tolerated the procedure well without complications. Patient was told to posture with face facing up.

At the postoperative day one visit, graft dislocation was noted (figure 1). Patient was sleeping on his side and not maintaining supine position during sleeping. He underwent additional intracameral air injections in order to achieve graft reattachment. However, the donor disc suddenly dislocated in the posterior chamber during injection of air. By May 2018 (two months after surgery), it was noted that the cornea had started to clear despite loss of the graft tissue. Uncorrected distance visual acuity had improved to 6/48 despite the donor lenticule graft being completely detached into posterior chamber (figure 2). His last follow up was in August 2018 and his cornea remained relatively clear. The postoperative treatment regime consisted of Dexamethasone $0.1 \%$ eye drops (MAXIDEX) three-hourly and Levofloxacin 0.5\% (CRAVIT) 4 times daily for 3 weeks and then Dexamethasone $0.1 \%$ eye drops (MAXIDEX) was tapered to 3 times daily for 1 week and then 2 times daily for 2 months and once a day for 1 month. At 3 months, the sutures were removed.

\section{DISCUSSION}

This report describes a case of spontaneous corneal clearance despite dislocated graft after Descemet Stripping Automated Endothelial Keratoplasty (DSAEK). Instead of a repeat keratoplasty, endothelial cells migration and repopulation has occurred resulting in corneal clearing and improved visual acuity in a patient with bullous keratopathy.

In DSAEK, the donor lamella consisting of functional endothelium, Descemet membrane (DM) and posterior stroma are placed onto the posterior surface of the recipient cornea to restore the normal cornea anatomy, and resume the endothelial function which is to pump out fluid from the cornea. Detachment of the donor tissue leads to oedema of the exposed recipient stroma and leads to graft failure.

Despite the anticipated pump function of the graft, there were few reported cases of spontaneous corneal clearance in the presence of graft detachment. Balachandran et al in 2009 reported cases of corneal clearing with improved visual acuity in 2 eyes of 2 patients with Fuchs' endothelial dystrophy after Descemet membrane endothelial keratoplasty (DMEK), despite early donor detachment. ${ }^{6}$ They proposed that in one case, the recipient endothelium may have migrated from the
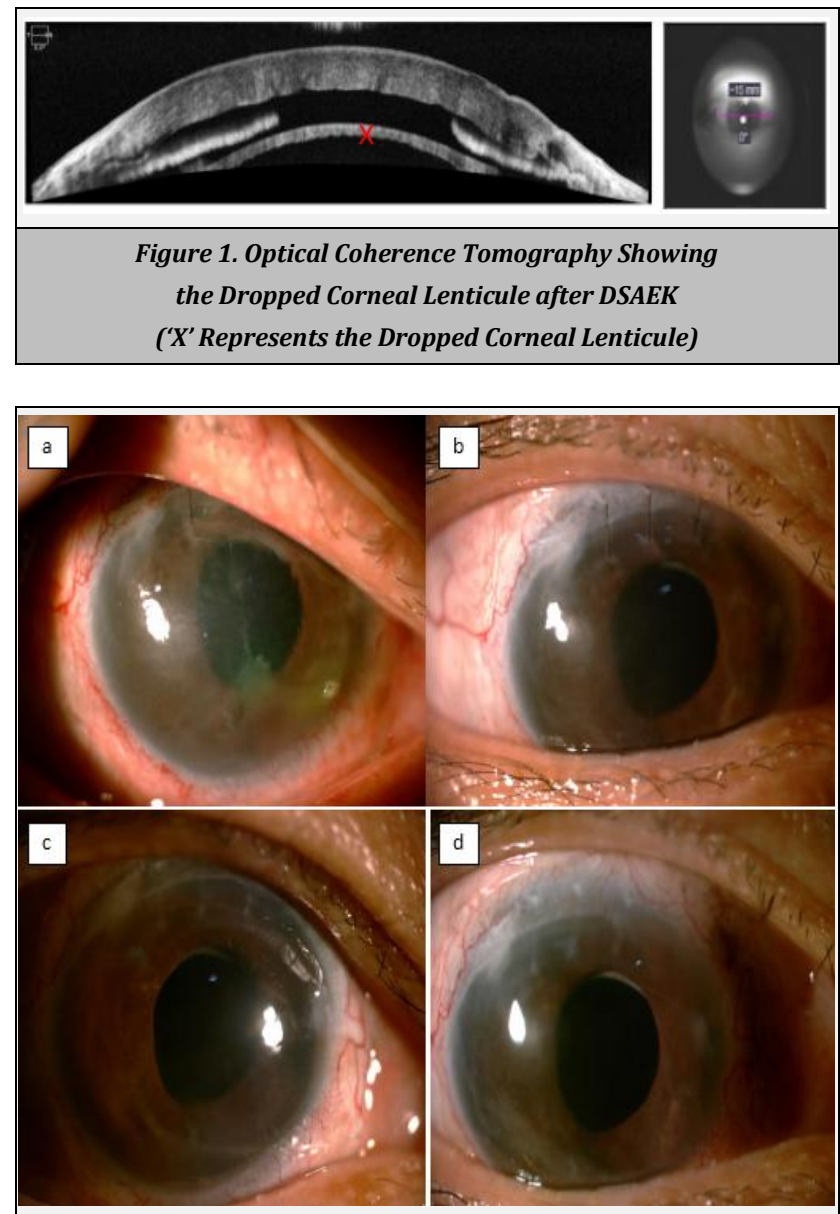

Figure 2. Post-Operative Anterior Segment Photos. Note That the Patients's Cornea Progressively Improves in Clarity. (a= Post-Op Week 1, b= Post Op Week 12, c= Post Op Week 14, d= Post Op Week 18)

remaining peripheral rim of recipient Descemet's membrane onto the bare recipient posterior stroma, from the area of the descemetorhexis. A second proposed mechanism was that an upside-down graft could have facilitated transfer of donor endothelial cells to bare host stroma. Shah et al in 2012 also reported a case of spontaneous corneal clearing after Descemet's stripping without endothelial replacement in their patient with a dual diagnosis of Fuchs' endothelial dystrophy and posterior polymorphous membrane dystrophy. ${ }^{9}$ Again, they proposed that host endothelial migration could be the possible mechanism for the central endothelial cell repopulation. Zafirakis et al in 2010 also reported a case of a clear cornea despite corneal graft detachment 9 months after DSAEK. ${ }^{5}$

In our patient, who was initially planned for traditional DSAEK, unfortunately a dislocated graft occurred. With the cornea clearing and improve visual outcome after about 5 months post-surgery without graft replacement, this might be due to the theory of endothelium migration from the remaining peripheral rim of Descemet's membrane onto the bare recipient posterior stroma, resulting in host endothelial cell repopulation. Visual acuity of $6 / 48$ was the best visual acuity our patient could achieve. This could be due to the presence of maculopathy from being highly myopic. Specular microscopy failed to detect any cells posterior to the cornea.

To prove the previous hypothesis, a study conducted by Isabel Bleyen et al in 2013 to determine whether the remaining endothelial cells from the clear peripheral rim 
would migrate and repopulate the center. ${ }^{12}$ However, from the study, they found that Descemet's stripping alone without endothelial keratoplasty was insufficient in patients with Fuchs' endothelial dystrophy. Further studies were suggested, including genetic analysis, to determine selection of patients who might benefit from Descemet's stripping alone.

In summary, spontaneous corneal clearance after DSAEK can occur without graft replacement but this applied to certain criteria of the patients. Age of the patient as well as cornea comorbidity are the important factors to determine its success.

\section{REFERENCES}

[1] American Academy of Ophthalmology (eyewiki.aao.org)

[2] Dapena I, Ham L, Melles GRJ. Endothelial keratoplasty: DSEK/DSAEK or DMAEK- the thinner the better? Curr Opin Ophthalmol 2009;20(4):299-307.

[3] Cursiefen C. Descemet membrane endothelial keratoplasty: the taming of the shrew. JAMA Ophthalmol 2013;131(1):88-9.

[4] Melles GRJ. Posterior lamellar keratoplasty: DLEK to DSEK to DMEK. Cornea 2006;25(8):879-81.

[5] Zafirakis P, Kymionis GD, Grentzelos MA, et al. Corneal graft detachment without corneal edema after descemet stripping automated endothelial keratoplasty. Cornea 2010;29(4):456-8.
[6] Balachandran C, Ham L, Verschoor CA, et al. Spontaneous corneal clearance despite graft detachement in descemet membrane endothelial keratoplasty. Am J Ophthalmol 2009;148(2):227-34.e1.

[7] Ham L, Dapena I, Moutsouris K, et al. Persistent corneal edema after descemetorhexis without corneal graft implantation in a case of Fuchs endothelial dystrophy, Cornea 2011;30(2):248-9.

[8] Arbelaez JG, Price MO, Price FW Jr. Long-term follow-up and complications of stripping descemet membrane without placement of graft in eyes with fuchs endothelial dystrophy. Cornea 2014;33(12):1295-9.

[9] Shah RD, Randleman BJ, Grossniklaus HE. Spontaneous corneal clearance after descemet's stripping without endothelial replacement. Ophthalmology 2012;119(2):256-60.

[10] Dirisamer M, Ham L, Dapena I, et al. Descemet membrane endothelial transfer: 'free-floating' donor descemet implantation as a potential alternative to 'keratoplasty'. Cornea 2012;31(2):194-7.

[11] Melles GRJ, Wijdh RHJ, Nieuwendaal CP. A technique to excise the descemet membrane from a recipient cornea (descemetorhexis). Cornea 2004;23(3):286-8.

[12] Bleyen I, Saelens IEY, van Dooren BTH, et al. Spontaneous corneal clearing after descemet's stripping. Ophthalmology 2013;120(1):215. 